\title{
Digitalisierung von Management-Reporting-Prozessen - Ein technologieorientiertes Reifegradmodell zum Einsatz in KMU
}

\author{
Pascal Stoffers • Jürgen Karla 1 · Jens Kaufmann $(i)$
}

Eingegangen: 12. April 2021 / Angenommen: 30. August 2021 / Online publiziert: 27. September 2021

(C) Der/die Autor(en) 2021

Zusammenfassung Der Begriff der Digitalisierung umfasst eine große Bandbreite unterschiedlicher Themen und Aktivitäten. Zur gezielten Entwicklung von Unternehmen ist es daher erforderlich, die eigene Position und entsprechende Verbesserungspotenziale zu (er)kennen und eine erfolgreiche Prozess-Weiterentwicklung zu ermöglichen. Das Controlling eines Unternehmens nimmt als Informationslieferant und Bestandteil der Entscheidungsunterstützung in vielfältiger Hinsicht Einfluss auf bestehende und zu entwickelnde Geschäftsmodelle und ist von der Digitalisierung besonders betroffen, insbesondere hinsichtlich der Planungs- und Reporting-Prozesse.

Zur Unterstützung der Digitalisierung vornehmlich in kleinen und mittleren Unternehmen wird ein Reifegradmodell entwickelt, das sich der beschriebenen Thematik widmet. Eine umfangreiche Vorstudie zeigt, welche existierenden Reifegradmodelle im Bereich der Managementinformation und des Controllings existieren und inwiefern diese noch nicht für die Fragestellung und/oder Anwendung ausreichend sind. Basierend auf der Identifikation von aktuellen, relevanten technologischen Entwicklungen werden Reifegradausprägungen eines neuen Modells erstellt, die die unterschiedlichen Dimensionen der unternehmerischen Tätigkeit einbeziehen.

In diesem Beitrag wird das Modell zur Bestimmung des aktuellen Reifegrads von Unternehmen entwickelt und vorgestellt.

\footnotetext{
Pascal Stoffers · Jürgen Karla $\cdot$ Jens Kaufmann $(\bowtie)$

Fachbereich Wirtschaftswissenschaften, Hochschule Niederrhein, Mönchengladbach, Deutschland

E-Mail: jens.kaufmann@hs-niederrhein.de

Pascal Stoffers

E-Mail: p.stoffers@ outlook.com

Jürgen Karla

E-Mail: juergen.karla@hs-niederrhein.de
} 
Schlüsselwörter Controlling · Digitalisierung $\cdot$ KMU $\cdot$ Management Reporting · Reifegradmodell

\section{Digitalizing Management Reporting Processes - A SME-Ready, Technology-Oriented Maturity Model}

Abstract Digitalization encompasses many different topics and activities. To foster a targeted development of companies, it is necessary to identify one's own position and optimization potentials. Only then can any process improvement be successful. The controlling unit of any company is considered to be both a source for information and a partner in the decision-making process for the management. Thus, it has a multi-faceted impact on business models and is considerably influenced by changes through digitalization, especially regarding planning and reporting processes.

To support digitalization, especially in small and medium enterprises, we present a maturity model that focuses on these topics. In a comprehensive pre-study, we show which maturity models already exist in the given area of management information and controlling and reason why they are not a complete fit to the purpose described. Based on current technological developments, we develop characteristics for a new model that incorporates different dimensions of a company's daily business.

In this paper, the model for determining the current maturity level of companies is developed and presented.

Keywords Controlling $\cdot$ Digitalization $\cdot$ Management Reporting $\cdot$ Maturity Model $\cdot$ SME

\section{Einleitung}

Der Begriff „Digitalisierung“ umfasst eine große Bandbreite an möglichen Themengebieten entlang der kompletten Wertschöpfungskette. Rund drei Viertel der deutschen Unternehmen verfolgen hierzu bereits eine Digitalstrategie, entweder in ausgewählten Unternehmensbereichen oder zentralisiert (BITKOM 2018). Im Controlling, das in besonderem Maße von der Digitalisierung betroffen ist, stehen nicht nur die Hauptfunktionen des Controllings als Informationslieferant und als Teil der Entscheidungsunterstützung im Blick (hier insbesondere die Planungs- und Reporting-Prozesse), sondern auch das Controlling-Verständnis als solches, wie aktuelle Beiträge, die die Digitalisierung als ein Kernthema des Controllings beschreiben, zeigen (Schäffer und Weber 2016; FINANCE 2019).

Durch die große Bandbreite des Themenkomplexes Digitalisierung in Kombination mit kontinuierlicher Weiterentwicklung und neuen Herausforderungen ist es für Unternehmen notwendig, den eigenen Entwicklungsstand sowie mögliches Verbesserungspotential zu (er)kennen. Dies ist grundlegend für eine erfolgreiche (Prozess-)Weiterentwicklung, da nur so im nächsten Schritt bestmöglich passende Ansätze verfolgt werden können (Kamprath 2011). Für kleine und mittlere Unternehmen (KMU), deren Strukturen typischerweise eben nicht denen von Konzernen mit spezialisierten Abteilungen entsprechen, ist dabei eine überbordende Komplexi- 
tät zu vermeiden. In diesem Beitrag wird daher ein Reifegradmodell für den in seiner Bedeutung steigenden Bereich des Management Reportings vorgestellt. Grundsätzlich handelt es sich dabei um ein generisches und deskriptives Modell, was aufgrund seiner bewusst niedrig gehaltenen Komplexität insbesondere für den Einsatz in KMU dienlich sein kann. Stützend für diese Motivation ist der allgemeine Bedarf von KMU nach Reifegradmodellen im Kontext der digitalen Transformation (Hölzle et al. 2019).

Der vorliegende Beitrag stellt in Abschn. 2 zunächst den maßgeblichen Einfluss von Digitalisierungstechnologien auf Management-Reporting-Prozesse vor und beschreibt die entstehenden Veränderungsmöglichkeiten und Potenziale. Abschn. 3 geht kurz auf die methodische Entwicklung von Reifegradmodellen ein. Die Entwicklung des in diesem Beitrag beschriebenen Reifegradmodells wird in Abschn. 4 systematisch dargelegt, indem bestehende Modelle identifiziert und bewertet sowie Dimensionen, Reifegrade und deren Zusammenführung und Operationalisierung (Abschn. 5) beschrieben werden. Der Beitrag schließt mit einer zusammenfassenden Darstellung der Erkenntnisse.

\section{Einfluss von Digitalisierungstechnologien auf Management- Reporting-Prozesse}

Die Anzahl und Varietät von Digitalisierungsansätzen sind groß. Burrus und Hood (2020) nennen als wichtigste vier Technologien im Bereich der Wirtschaftsprüfung „Big Data“, „Künstliche Intelligenz“, „Cloud Computing“ und „Robotic Process Automation“ (RPA). Diese werden im Folgenden kurz vorgestellt, um ihren Einfluss auf Management-Reporting-Prozesse (MRP) zu erläutern. Ergänzend wird mit „Business Analytics“ ein Thema beleuchtet, das sich unmittelbar mit der Anwendung analytischer Verfahren zur Datenauswertung befasst (Felden 2019).

\subsection{Ausgewählte Digitalisierungstechnologien}

In Unternehmen besteht eine hohe Bereitschaft zu Investitionen in Technologien und Lösungen rund um Big Data (KPMG 2018). Zur Definition des Begriffs werden üblicherweise mehrere Eigenschaften miteinander kombiniert, die z. B. im 5V-Modell Ausdruck finden und Big Data kennzeichnen als geprägt von „Volume“ (Datenmenge), ,Variety“ (Unterschiedlichkeit), ,Velocity“ (Entstehungs- und Veränderungsgeschwindigkeit) sowie ergänzend „Value“ (Wert der Daten für das Unternehmen) und „Veracity“ (Wahrhaftigkeit) (Fasel und Meier 2016).

Künstliche Intelligenz (KI) kann im Sinne dieses Artikels als eine Technik zur Nachbildung menschlicher Intelligenz mit dem Fokus auf Lernen sowie der Wahrnehmung und dem Verständnis von Zusammenhängen aufgefasst werden. „Schwache KI“" ist dabei auf spezielle Probleme fokussiert, während eine ,starke KI“ allgemeine Intelligenz zeigt und eventuell sogar menschliche Fähigkeiten übertreffen soll (Kirste und Schürholz 2019).

Cloud Computing kann als digitale Infrastrukturalternative zu klassischen OnPremise- oder Hosted-Lösungen gesehen werden, bei denen der Kunde bzw. das 
entsprechende Unternehmen die volle Kontrolle über das System und die Daten behält. Bei Cloud Computing hingegen wird ein (ausprägungsabhängig großer) Anteil der IT-Infrastruktur-, Plattform- und Service-Dienstleistungen an Externe übergeben.

Bei RPA handelt es sich um Software, die strukturierte Routineaufgaben zur $\mathrm{Au}-$ tomatisierung von Prozessschritten über verschiedene Systeme hinweg übernimmt. Dabei werden die Handlungen des Menschen imitiert. Grafische Benutzeroberflächen gestalten den Konfigurationsprozess vergleichsweise einfach. In den letzten Jahren haben RPA-Lösungen kontinuierlich an Beliebtheit gewonnen. Dieser Trend wird auch durch Berichte von einem hohen Return on Investment bei RPA-Projekten oder von geringen Kosten für die Einführung begünstigt (Willcocks et al. 2015; Langmann und Turi 2020).

Business Analytics (BA) ist ein weiterer Schlüsselbegriff der Digitalisierung. BA „kann als Sammlung unterschiedlicher Methoden und Technologien verstanden werden, welche dazu dienen, Erkenntnisse aus verfügbaren Daten für unternehmerische Entscheidungen zur Steuerung der Geschäftsprozesse zu gewinnen." (Chamoni und Gluchowski 2017). Im Analytics-Bereich werden häufig verschiedene, inhaltlich überlappende Begriffe verwendet. Speziell „Business Intelligence“ (BI) gilt als Sammelbegriff für unterschiedliche Aktivitäten im Bereich Datenanalyse.

\subsection{Management-Reporting-Prozesse}

Reporting nimmt in Unternehmen eine wichtige Stellung ein, da die berichteten Informationen essenziell für Entscheidungsprozesse sind. Ihm kommt damit eine der bedeutsamsten Aufgaben im Controlling zu (Taschner 2019). Unter Reporting ist im weitesten Sinne ,die Informationsbedarfsermittlung, -beschaffung, -aufbereitung, -bereitstellung, -nutzung und -analyse aller steuerungs- und entscheidungsrelevanter Informationen des Betriebs und seiner Umwelt für externe und interne Adressaten des Unternehmens in Form von Berichten zu verstehen, wobei diese idealerweise adressatengerecht gebündelt in einem Reporting-System aufbereitet werden.“ (Schön 2018). Reporting, als eine der Hauptaufgaben des Controllings, folgt daher in jedem Unternehmen üblicherweise einem eigenen, speziellen Prozess. Dem Management Reporting (MR) liegt ein Prozess zugrunde, welcher im weitesten Sinne bei der Informationsbedarfsermittlung startet und bei der Informationsverwendung endet. Je nach Anforderungen und Ressourcen des Unternehmens sind die einzelnen Prozessschritte verschieden stark ausgeprägt, zeitaufwändig und von unterschiedlicher Qualität im Hinblick auf Effizienz, Schnelligkeit und Korrektheit. Unabhängig von den Anforderungen ist der Prozess an sich jedoch über die verschiedensten Unternehmen und Branchen hinweg allgemein vergleichbar, da das Ziel der Informationsverteilung universell gilt. Basierend auf Taschner (2013) und Gräf et al. (2014) lässt sich beispielhaft der in Abb. 1 dargestellte Prozess mit Unterschritten synthetisieren.

Zunächst ist für die Bereitstellung der passenden Daten eine Bedarfsermittlung notwendig. Darauf basierend werden geeignete interne oder auch externe Datenquellen identifiziert, die die benötigten Daten liefern können. Nach der Identifikation der Quellen werden entsprechende Abfragen eingerichtet, um tatsächlichen Zugriff auf die Daten zu bekommen. Zuletzt werden die gesammelten Daten aufbereitet. Dies 

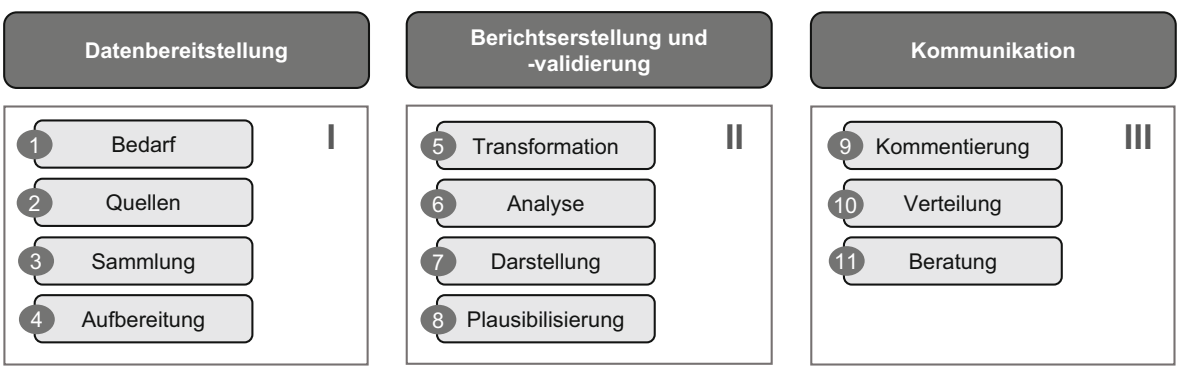

Abb. 1 Schritte im Management-Reporting-Prozess (in Anlehnung an Taschner (2013) und Gräf et al. (2014))

umfasst die Prüfung auf Vollständigkeit, fehlende oder falsche Einträge sowie die allgemeine Konsistenz der Daten.

Die anschließende Transformation beinhaltet notwendige Umformungen, Aggregationen, Aufspaltungen oder Verbindungen, damit im nächsten Schritt zuerst einfache Kennzahlen berechnet werden können und dann tiefergehende Analysen durchgeführt werden können. Es wird nach Ursachen und Kausalketten gesucht, um Sachverhalte fundiert erklären oder Vorhersagen treffen zu können. Nach der Analyse werden die Ergebnisse dargestellt und plausibilisiert.

Basierend auf den Resultaten der Analyse wird das Reporting kommentiert, um so Zusammenhänge zu verdeutlichen und wichtige Entwicklungen in den Vordergrund zu rücken. Abschließend werden die gesammelten Ergebnisse inkl. Kommentaren an die Empfänger verteilt. Bei Rückfragen werden Gespräche geführt und weitere Schritte erarbeitet. Letztendlich sollen die Daten von den Empfängern entsprechend genutzt und interpretiert werden, um bei der Umsetzung von Vorschlägen und bei Entscheidungen zu unterstützen. Dies ist der ausschlaggebende, beratende Aspekt des MR.

\subsection{Digitalisierungstechnologien in einzelnen Prozessschritten}

Die vorgestellten Digitalisierungstechnologien wirken auf unterschiedliche Stellen des gesamten Management-Reporting-Prozesses.

Big Data zeichnet sich in erster Linie dadurch aus, eine weitaus breitere Zahl an Quellen mit größeren Datenmengen zu bieten. Es entstehen aussagekräftigere Informationen durch eine erhöhte Aktualität und die Möglichkeit unternehmensinterne Daten wie z. B. Umsatzzahlen mit externen Daten, z.B. aus sozialen Medien, zu kombinieren (Weichel und Herrmann 2016). Daraus können z.B. Frühwarnsysteme, verbesserte Prognosen oder dynamische KPIs resultieren (Dursun 2019). Auch rein technisch können Big-Data-Methoden geeignet sein, die Datensammlung und -verarbeitung im MR zu beschleunigen.

KI kann verwendet werden, um in Datenmengen Muster zu entdecken oder die Datenqualität zu verbessern. Letzteres kann geschehen, indem z. B. händisch in ein Quellsystem eingetragene Werte sofort auf Plausibilität überprüft werden. Neben dieser Rolle von KI als „Qualitätsprüfer“ ist auch eine Anwendung im Bereich Verteilung und Beratung als digitale Assistenten möglich (Friedl 2019). 
Cloud Computing macht einige Verbesserungen im Zuge der Digitalisierung überhaupt erst möglich und beeinflusst den MRP von der Speicherung der Daten bis hin zur Verteilung der Informationen. Durch eine einfache und wirtschaftliche Bereitstellung von Infrastruktur können Analysen mit einem höheren Detailgrad durchgeführt werden. Die höhere Verfügbarkeit unterstützt Self-Service-BI-Systeme und Mobile Reporting.

RPA hat insbesondere das Potenzial, die Prozessschritte im Bereich Datenbereitstellung sowie Berichtserstellung und -validierung zu automatisieren. Diese Art von Automatisierung verringert Durchlaufzeiten und Fehlerquoten. So können sich Controller auf wertschöpfende Tätigkeiten wie Beratung konzentrieren - ein Effekt, der sich auf allgemeiner Ebene bereits gezeigt hat (Forrester 2018).

Business Analytics beschäftigt sich grundsätzlich mit Methoden zur Gewinnung von entscheidungsrelevanten Informationen. So können z.B. statistische Methoden unkonventionelle Korrelationen bzw. Wertetreiber aufdecken und so Entscheidungen beeinflussen. Mit „Predictive Analytics“ lassen sich Analysen erstellen, die darauf fokussieren, neue Beobachtungen vorherzusagen (Shmueli und Koppius 2011). Für das Reporting bedeutet dies, dass neuartige Informationen verfügbar werden, welche Entscheidungen in der Gegenwart beeinflussen.

\subsection{Besonderheiten bei kleinen und mittleren Unternehmen}

Kleine und mittlere Unternehmen machten im Jahr 2017 in Deutschland ca. 99,3\% aller Unternehmen aus, 60,8\% aller Beschäftigten arbeiten in KMU und diese haben einen Anteil von 33,2\% am gesamten Unternehmensumsatz in Deutschland (Statistisches Bundesamt (Destatis) 2020). Die Unternehmensgröße hat zur Folge, dass begrenzte Ressourcen verfügbar sind, was KMU kompensieren oder anderweitig berücksichtigen müssen (De Massis et al. 2018). Auf das Reporting bezogen führt dies ggf. zu einem anderen Stellenwert und zu anderen Zielen. Einerseits zeigt sich dies in einem geringeren Detailgrad (Hauptsächlich finanzielle Kennzahlen, wenig bis keine Einteilung in Sparten bzw. Kostenträger, keine Nutzung von externen Informationen) und damit vermeintlich auch in geringerer Aussagekraft des MR. Andererseits sind die MRP häufiger historisch gewachsen, es liegt also kein ganzheitliches Konzept vor (Schön 2012). Die Konsequenz daraus kann z. B. eine fragmentierte Softwarelandschaft sein, welche wiederum zu einem aufwändigeren Prozess führt, da zusätzliche Schritte in den Bereichen Datensammlung, -aufbereitung und -transformation notwendig sind. Besonderheiten von KMU müssen in der Arbeit entsprechend berücksichtigt werden, so wie es auch bei der Entwicklung von allgemeineren Reifegradmodellen zur digitalen Transformation von KMU der Fall ist (Hölzle et al. 2019).

\section{Methodische Entwicklung von Reifegradmodellen}

Reifegradmodelle sind ein Instrument zur Standortbestimmung eines betrachteten Objekts und zeigen einen möglichen oder typischen Entwicklungspfad für dieses auf (Becker et al. 2009). Dieses Objekt kann z.B. ein Prozess, eine Organisation oder 


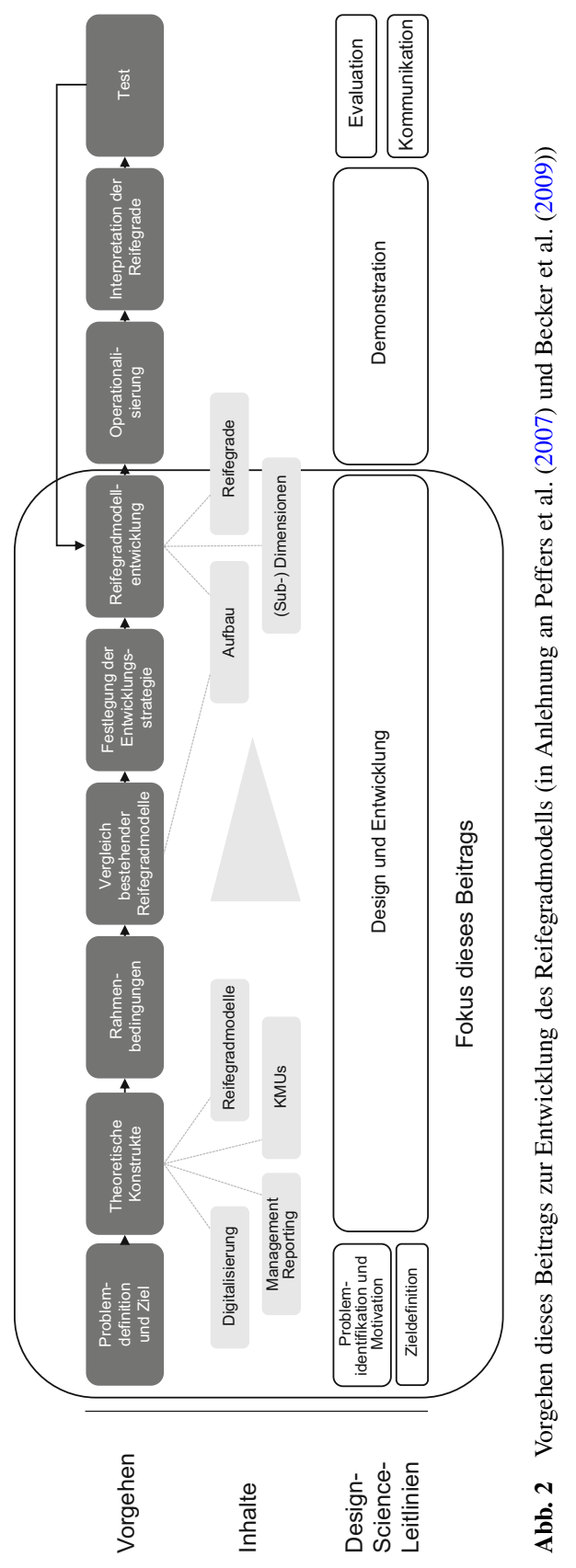


ein Projekt sein. Dabei wird die eigene ermittelte Reife (Kompetenz, Möglichkeiten, Komplexität) mit internen oder externen vorgegebenen Referenzgrößen verglichen. Das Ergebnis dieser Bewertung dient dann als Grundlage zur Verbesserung und Fortschrittskontrolle des eingeordneten Objekts (de Bruin et al. 2005). Generell ist ein Reifegrad durch verschiedene Merkmale, welche wiederum in Dimensionen aufgeteilt sein können, definiert. Um die spezifischen Merkmalsausprägungen in Unternehmen zu bestimmen, werden Bewertungsmethoden wie z. B. Fragebögen verwendet (Becker et al., 2009).

Im Sinne des Design-Science-Research schlagen Peffers et al. (2007) eine allgemeine Methode basierend auf den Richtlinien von Hevner et al. (2004) vor, um Artefakte zu konstruieren, zu denen auch ein Reifegradmodell gehören kann. Eine mögliche Vorgehensweise für Reifegradmodelle in sechs Phasen geben de Bruin et al. (2005) vor, eine alternative Variante von acht Schritten, die mit dem genannten Design-Science-Ansatz eng verzahnt ist, findet sich bei Becker et al. (2009).

Beide vorgestellten Vorgehensweisen zeigen einen strukturierten Weg zur Entwicklung eines Reifegradmodells auf, betonen allerdings unterschiedliche Teilbereiche. Aufgrund der engen Verbindung mit dem Design-Science-Ansatz und der detaillierten Ausarbeitung des Vorgehens, basiert die in diesem Beitrag verwendete Vorgehensweise zu einem großen Teil auf dem Vorgehensmodell von Becker et al. (2009). Zur Vorbereitung des Vergleichs mit bestehenden Modellen wird zusätzlich die Klärung der Rahmenbedingungen eingefügt, in Anlehnung an die Schritte „Scope“ und (in Teilen) „Design“ von de Bruin et al. (2005). Becker et al. (2009) unterscheiden zwischen der Konzeption von Transfer und dem Transfer an sich. In diesem Beitrag wird der Schritt als „Operationalisierung“ abgebildet und beschäftigt sich mit der Übertragung des Modells in einen praktisch anwendbaren Fragebogen. Die erste Kommunikation fand im Anschluss im Rahmen des Tests statt. Vor diesem Test, der damit einer verkürzten Form der Evaluation entspricht, wird in Anlehnung an Pöppelbuß und Röglinger (2011) eine Interpretation der Reifegrade durchgeführt. Im Fokus dieses Beitrags stehen allerdings Design und Entwicklung des Modells.

Abb. 2 stellt Vorgehen und Verknüpfung zu den Design-Science-Prinzipien sowie den fachlichen Inhalten dieses Beitrags zusammenfassend dar.

\section{Entwicklung eines Reifegradmodells zur Digitalisierung von Management-Reporting-Prozessen}

In diesem Abschnitt werden zunächst die Rahmenbedingungen festgelegt und dann ein Vergleich bestehender Modelle durchgeführt. Mit Aufbau, Dimensionen und Reifegraden werden die Bestandteile des Reifegradmodells entlang der skizzierten Entwicklungsstrategie erarbeitet, sodass abschließend ein vollständiges Modell entsteht.

\subsection{Rahmenbedingungen}

Das hier entwickelte Modell ist Domänen-spezifisch mit einem Fokus auf Management Reporting. Es hat zwar einen akademischen Ursprung, ist aber zur internen 
Nutzung für Fach- und Führungskräfte aus dem Bereich Controlling gedacht, mit dem Ziel den Stand der Digitalisierung der MR-Prozesse zu ermitteln und Entwicklungspfade aufzuzeigen. Eine externe Nutzung durch z. B. Unternehmensberatungen zur Diagnostik ist denkbar. Das Modell kann - obschon deskriptiver Natur - keinen Status Quo der Digitalisierungsbemühungen in Deutschland insgesamt liefern, hierzu sei z.B. auf Büchel et al. (2021), Deutsche Telekom (2020) oder Grzymek und Wintermann (2020) verwiesen.

Die Anwendung erfolgt durch eine Selbstbewertung mit Hilfe eines Fragebogens. Falls den angesprochenen Fachkräften ein IT-Hintergrund fehlt, empfiehlt es sich, die Bewertung zusammen mit IT-Fachkräften auszufüllen. Unternehmen bzw. Gruppen, in denen Konsolidierungsaufgaben einen großen Teil des Reportings ausmachen, sind nicht die primäre Zielgruppe dieses Modells, da deren Reporting-Prozess leicht von den bisherigen Beschreibungen abweicht. Das Modell wird primär für Einzelunternehmen oder Teile von Gruppen mit eigenem Reporting entworfen. Der Fokus liegt zwar auf KMU, dies zeigt sich aber besonders bei der Interpretation des Modells. Die Standortbestimmung an sich können auch größere Unternehmen ohne Probleme vornehmen, solange die restlichen Rahmenbedingungen erfüllt werden.

\subsection{Vergleich bestehender Modelle}

Als relevant für den Vergleich werden Werke betrachtet, in denen Reifegradmodelle erarbeitet bzw. neu vorgestellt werden. Der Fokus liegt dabei auf Werken zum Management Reporting, ergänzend auf Beiträgen zum Controlling. Weniger relevant, aber der Vollständigkeit halber mit betrachtet sind Veröffentlichungen zu den Themen BA bzw. BI, sowohl wegen der möglichen thematischen und begrifflichen Überschneidung als auch zur Behandlung anderer Digitalisierungstechnologien wie z. B. Big Data. Reifegradmodelle zum Thema „Business Process Management“ hingegen werden als zu allgemein angesehen, um hier umfassend mit einbezogen zu werden.

Mit Hilfe eines mehrstufigen Verfahrens zur Literaturrecherche, basierend auf vom Brocke et al. (2009) und Webster und Watson (2002), wurden zunächst über die Datenbanken EBSCOhost (business/academic source premier), SpringerLink, Wiso, Web of Science, Science Direct, JSTOR und Google Scholar 44 relevante Publikationen durch Titel- und Abstract-Prüfung identifiziert. Mit einer vollständigen Inhaltsprüfung wurde in einer zweiten Sichtung die Anzahl auf 11 reduziert, mit denen nachfolgend wiederum eine Rückwärtssuche durchgeführt wurde, sodass 13 Modelle abschließend als relevant identifiziert wurden (Ausführliches Listing in Onlinematerial 1). Tab. 1 stellt diese Modelle, die darin vermerkten Reifegrade sowie Dimensionen und eine vorgenommene Kategorisierung dar.

In der abschließenden Betrachtung der Modelle zeigt sich, dass keines bereits ausreichend fachlich fokussiert oder die Rahmenbedingungen, die als Motivation zur Erarbeitung des Modells bestimmt wurden, ausreichend berücksichtigt. Sofern in der bestehenden Literatur Management Reporting ausdrücklich betrachtet wurde, lag der Fokus der Modelle nicht auf einer expliziten Einbindung von Kriterien für Digitalisierungstechnologien. Diese stellen der obigen Analyse nach jedoch einen wesentlichen Treiber der Digitalisierung in Unternehmen dar und sollten entspre- 
Tab. 1 Ergebnis der Literaturrecherche zu relevanten Reifegradmodellen

\begin{tabular}{|c|c|c|c|}
\hline Modell & Dimensionen & Reifegrade & Kategorie \\
\hline $\begin{array}{l}\text { USS-Reifegrad- } \\
\text { modell } \\
\text { (Marx et al. } \\
\text { 2012) }\end{array}$ & $\begin{array}{l}\text { Berichterstattung, Planung, } \\
\text { Konsolidierung }\end{array}$ & $\begin{array}{l}\text { Grundlegender Ansatz, Ausge- } \\
\text { wogener Ansatz, Umfassender } \\
\text { Ansatz, Strategieorientierter } \\
\text { Ansatz, BI-gestützter Ansatz }\end{array}$ & $\begin{array}{l}\text { MR- } \\
\text { orientiert }\end{array}$ \\
\hline $\begin{array}{l}\text { MIS Reifegrad- } \\
\text { modell } \\
\text { (Schulze und } \\
\text { Watling 2013) }\end{array}$ & $\begin{array}{l}\text { Verantwortlich, Medien, Scope, } \\
\text { Charakteristikum }\end{array}$ & $\begin{array}{l}\text { Anarchie, Nische, Expansion, } \\
\text { Standard, Service }\end{array}$ & $\begin{array}{l}\text { MR- } \\
\text { orientiert }\end{array}$ \\
\hline $\begin{array}{l}\text { Reifegradmodell } \\
\text { des digitalen } \\
\text { Controllings } \\
\text { (Koß 2016) }\end{array}$ & - & $\begin{array}{l}\text { Insellösungen, integriertes } \\
\text { System, digitale Identität, } \\
\text { digitales Ökosystem }\end{array}$ & $\begin{array}{l}\text { Controlling } \\
\text { orientiert }\end{array}$ \\
\hline $\begin{array}{l}\text { Reifegradmodell } \\
\text { für die Digita- } \\
\text { lisierung des } \\
\text { Controllings } \\
\text { (Langmann } \\
\text { 2019) }\end{array}$ & $\begin{array}{l}\text { Rollenmodell, Organisation, } \\
\text { Prozesse, Governance, IT-Sys- } \\
\text { tem, Personal \& Kompetenzen }\end{array}$ & $\begin{array}{l}\text { Digital Newbie, Digital Main- } \\
\text { stream, Digital Pioneer }\end{array}$ & $\begin{array}{l}\text { Controlling } \\
\text { orientiert }\end{array}$ \\
\hline $\begin{array}{l}\text { DigiCon } \\
\text { (Keimer et al. } \\
\text { 2017) }\end{array}$ & $\begin{array}{l}\text { Prozesse, Organisation, Da- } \\
\text { ten, Methoden, Technologien, } \\
\text { Kompetenzen }\end{array}$ & Stufen 1-5 & $\begin{array}{l}\text { Controlling } \\
\text { orientiert }\end{array}$ \\
\hline $\begin{array}{l}\text { Optimized BI } \\
\text { Maturity Model } \\
\text { (Tavallaei et al. } \\
\text { 2015) }\end{array}$ & $\begin{array}{l}\text { Structure and rules, Organiza- } \\
\text { tional Culture, Technology }\end{array}$ & $\begin{array}{l}\text { Initial, Immature, Controlled, } \\
\text { Managed, Mature }\end{array}$ & $\begin{array}{l}\text { BI- } \\
\text { orientiert }\end{array}$ \\
\hline $\begin{array}{l}\text { biMM: Business } \\
\text { Intelligence } \\
\text { Maturity Model } \\
\text { (Chamoni und } \\
\text { Gluchowski } \\
\text { 2004) }\end{array}$ & $\begin{array}{l}\text { Fachlichkeit, Technik, Organi- } \\
\text { sation }\end{array}$ & $\begin{array}{l}\text { Vordefiniertes Berichtswesen, } \\
\text { BI pro Fachbereich, Unter- } \\
\text { nehmensweite BI, Erweiterte } \\
\text { Entscheidungsunterstützung, } \\
\text { Aktives Wissensmanagement }\end{array}$ & $\begin{array}{l}\text { BI- } \\
\text { orientiert }\end{array}$ \\
\hline $\begin{array}{l}\text { EBIM: Enterprise } \\
\text { Business Intelli- } \\
\text { gence Maturity } \\
\text { (Tan et al. 2011) }\end{array}$ & $\begin{array}{l}\text { Information Quality, Master } \\
\text { Data Management, Warehou- } \\
\text { sing Architecture, Analytics }\end{array}$ & $\begin{array}{l}\text { Initial, Repeatable, Defined, } \\
\text { Managed, Optimized }\end{array}$ & $\begin{array}{l}\text { BI- } \\
\text { orientiert }\end{array}$ \\
\hline $\begin{array}{l}\text { LOBI: The lad- } \\
\text { der of Business } \\
\text { Intelligence } \\
\text { (Cates et al. } \\
\text { 2005) }\end{array}$ & - & $\begin{array}{l}\text { Facts, Data, Information, } \\
\text { Knowledge, Understanding } \\
\text { Enabled intuition }\end{array}$ & $\begin{array}{l}\text { BI- } \\
\text { orientiert }\end{array}$ \\
\hline $\begin{array}{l}\text { Coporate Perfor- } \\
\text { mance Manage- } \\
\text { ment Maturity } \\
\text { (Aho 2009) }\end{array}$ & $\begin{array}{l}\text { Management and Organisation, } \\
\text { Technology, People \& Culture, } \\
\text { Processes }\end{array}$ & $\begin{array}{l}\text { Unaware, Ad-hoc, Repeatable, } \\
\text { Defined, Managed, Optimized }\end{array}$ & $\begin{array}{l}\text { BI- } \\
\text { orientiert }\end{array}$ \\
\hline $\begin{array}{l}\text { TDWI Big Data } \\
\text { Maturity Model } \\
\text { (Halper und } \\
\text { Krishnan 2013) }\end{array}$ & $\begin{array}{l}\text { Infrastructure, Data Manage- } \\
\text { ment, Analytics, Governance, } \\
\text { Organization }\end{array}$ & $\begin{array}{l}\text { Nascent, Pre-Adoption, Early } \\
\text { Adoption, Corporate Adoption, } \\
\text { Mature }\end{array}$ & $\begin{array}{l}\text { Daten- } \\
\text { orientiert }\end{array}$ \\
\hline
\end{tabular}


Tab. 1 (Fortsetzung)

\begin{tabular}{|c|c|c|c|}
\hline Modell & Dimensionen & Reifegrade & Kategorie \\
\hline $\begin{array}{l}\text { DWCMM: Data } \\
\text { Warehouse ca- } \\
\text { pability maturity } \\
\text { model } \\
\text { (Spruit und Sacu } \\
2015 \text { ) }\end{array}$ & $\begin{array}{l}\text { Architecture, Data Modelling, } \\
\text { ETL, BI Applications, De- } \\
\text { velopment Process, Service } \\
\text { Processes }\end{array}$ & $\begin{array}{l}\text { Initial, Repeatable, Defined, } \\
\text { Managed, Optimized }\end{array}$ & $\begin{array}{l}\text { Daten- } \\
\text { orientiert }\end{array}$ \\
\hline $\begin{array}{l}\text { IT-Einsatz in } \\
\text { KMU } \\
\text { (Wagner 2012) }\end{array}$ & $\begin{array}{l}\text { Prozesse und Ressourcen, } \\
\text { Risiken und IT Sicherheit, } \\
\text { Mitarbeiter, Wertbeitrag der IT, } \\
\text { Prozesse und Ressourcen, IT- } \\
\text { Strategie und Governance }\end{array}$ & $\begin{array}{l}\text { Nicht existent, muss, sollte, } \\
\text { kann, exzellent }\end{array}$ & $\begin{array}{l}\text { Allgemein- } \\
\text { orientiert }\end{array}$ \\
\hline
\end{tabular}

chend fokussiert betrachtet werden. Die identifizierten Digitalisierungstechnologien werden außerdem in den nicht-MR-orientierten Reifegradmodellen nur punktuell adressiert. Bei der Erarbeitung von Inhalten und Strukturen für das Reifegradmodell dieses Beitrags sind aufgrund der thematischen Nähe, insbesondere die ersten beiden Kategorien (MR-orientiert, Controlling-orientiert) relevant.

\subsection{Dimensionen}

Es lässt sich festhalten, dass alle Modelle mit durchschnittlich vier Dimensionen und fünf Reifegraden arbeiten. Dabei sind die Dimensionen entweder allgemein gehalten und werden in der Bewertung bzw. mit Sub-Dimensionen weiter spezifiziert (z. B. beim biMM) oder die Dimensionen sind an sich schon spezifisch (z.B. beim DWCMM). Im Folgenden werden geeignete, durch die vorgestellten Aktivitäten im Management-Reporting-Prozess fundierte Dimensionen und Sub-Dimensionen abgeleitet und vorgestellt, wobei - sofern passend - die Erkenntnisse der relevanten betrachteten Modelle mit aufgenommen werden.

\subsubsection{Datenbereitstellung}

Die Datenbereitstellung ist grundlegend für die weiteren Dimensionen und damit Prozessschritte im MR. Die fünf Sub-Dimensionen dieser Dimension werden aus den theoretischen Konzepten, insbesondere zu Big Data, und den Erkenntnissen aus den bestehenden Modellen abgeleitet.

Datenumfang Die Verarbeitung großer Datenmengen im Sinne des Big-Data-Ansatzes bedarf entsprechender Prozesse und Technologien. Gemessen wird, ob nur wenige, interne, strukturierte Daten oder ergänzend auch externe, unstrukturierte Massendaten verwendet werden.

Datenspeicherung und -verarbeitung Im Optimalfall sind die Daten zentral und schnell bis hin zu real-time in Big-Data-konformen DWHs verfügbar. Im schlechtesten Fall besteht keine zentrale Datenbank oder ähnliches und es werden einzelne, lokal gespeicherte Datensätze verwendet (Spruit und Sacu 2015). Die Verarbeitung 
von großen, unstrukturierten Datenmengen ist dann nicht möglich und auch die Verarbeitungsgeschwindigkeit ist unter Umständen limitiert.

Datenqualität Nur ein konsistenter und anerkannter Datenbestand kann zu akzeptierten Berichten und Analysen führen (,Veracity“). Die Betrachtungen zur Datenqualität folgen üblichen Quellen, eine Zusammenstellung bietet beispielsweise Fürber (2016).

Datenverantwortlichkeit Eine dedizierte Data Governance betont, dass der Wert von Daten verstanden wurde. Zusätzlich werden Aspekte wie z. B. Datensicherheit oder Datenschutz abgedeckt.

Automatisierung der Datenbereitstellung Die Automatisierung der anfallenden Prozessschritte im Bereich Datenbereitstellung ist besonders im Bereich ,digitales Reporting“ ein wichtiges Thema (Isensee et al. 2019). Dabei ist volle Automatisierung erstrebenswert und manuelle Tätigkeiten sind bestmöglich zu vermeiden.

\subsubsection{Berichtserstellung und -validierung}

Das zentrale Element der Dimension Berichtserstellung und -validierung ist die Analyse der Daten. Die Digitalisierungstechnologien BA und KI stehen dabei im Mittelpunkt.

Analytische Anwendungen Diese reichen von generischer Software bis hin zu dedizierter, spezialisierter BI-Software mit Data-Warehouse- und Big-Data-Unterstützung. In niedrigen Reifegraden sind die Diversität und Qualität der Analysen durch die Anwendung von generischen Tabellenkalkulationsprogrammen oder einfachen integrierten Analysefunktion von ERP-Software eingeschränkt.

Analytische Methoden Angelehnt an die Reifegrade der Ladder of Business Intelligence (Cates et al. 2005) kann die Anwendung verschiedener Methoden entweder nur zu Informationen (deskriptiv) führen oder im ausgeprägtesten Stadium zu „Intuition“" (präskriptiv).

Darstellung Als einfachste Form sind einfache Tabellen und eine Dekoration von Informationen denkbar. Weiter fortgeschritten werden interaktive Dashboards mit OLAP-Funktionen verwendet. Besonders bei Analysen mit Big Data gibt es zusätzlich innovative Visualisierungen, welche eine verbesserte Informationsaufnahme bewirken können (Losbichler et al. 2016).

Plausibilisierung In der einfachsten Form finden manuelle Stichproben und die Nutzung von Erfahrungswerten Anwendung. Fortgeschrittene Formen setzen systematische Vorgehensweisen ein und eine weitergehende Digitalisierung führt zum Einsatz von KI-Methoden zur Datenvalidierung oder Fehlererkennung. 
Automatisierung der Analyse Die Betrachtung entspricht den Überlegungen der Dimension Datenbereitstellung, hier allerdings mit einem Fokus auf die Analysetechniken.

\subsubsection{Kommunikation}

In den Prozessen zur Kommentierung, Verteilung und Beratung der bereits dargestellten Analyseergebnisse liegt die eigentliche Wertschöpfung des MR. Entsprechend dem Credo „Die richtige Information am richtigen Ort zur richtigen Zeit“" sind die folgenden Sub-Dimensionen aufgebaut.

Medium Während Papier die einfachste und herkömmlichste Form der Informationsbereitstellung ist, bieten passive elektronische Verteilungsmechanismen, wie beispielsweise zentral abgelegte PDF-Dateien, eine einfachere und leichter parallelisierbare Auswertung. Noch flexibler sind (Cloud-gestützte) interaktive Zugriffe, die Self-Service-Lösungen und mobile Anzeigen ermöglichen.

Aktualität Die Bereitstellung von Informationen in Echtzeit kann als maximale Aktualität beschrieben werden. Für fundierte Analysen sollten außerdem so viele vergangene Daten wie möglich verwendbar sein. Abschwächungen dieser Varianten finden sich auf geringeren Reifegraden des Modells.

Kommentierungs- und Beratungsform In einer rudimentären Form werden Berichte nur kommentiert. Das Aufzeigen von Wirkungsketten und Kausalzusammenhängen stärkt die Interpretationsfähigkeit. Eine hochwertige und Fragen-orientierte Kommentierung kann durch Handlungsempfehlungen ergänzt werden (Holinski 2014). In einer noch stärker digitalisierten Form können interaktive Beratung, Storytelling und KI-gestützte Rückfragemöglichkeiten implementiert werden.

Automatisierung der Kommentierung und Beratung Die Betrachtung entspricht auch hier den Überlegungen der vorherigen Dimension.

\subsubsection{Umfeld}

Die Dimension Umfeld beschreibt Themen, die hauptsächlich indirekt auf den Digitalisierungsgrad des MR einwirken. Sie ist, wie die nachstehenden vier Sub-Dimensionen, größtenteils an die Vergleichs-Reifegradmodelle angelehnt.

Kompetenz der Mitarbeiter Fehlende Kenntnisse rund um die Bereiche Digitalisierung, Datenanalyse, Datenbanken, Statistik und Programmierung können sämtliche Digitalisierungsinitiativen entweder ausbremsen oder sogar komplett eindämmen (Keimer et al. 2017).

Rolle des Management Reportings im Unternehmen Abhängig von der Wahrnehmung und dem Nutzen des MR für das Unternehmen, werden z. B. mehr Ressour- 
cen zur Weiterentwicklung der MRP verfügbar gemacht, was dann eine Förderung von Digitalisierungsprojekten ermöglicht (Koß 2016).

Veränderungsbereitschaft aller Beteiligten am MR-Prozess Im Gegensatz zu den anderen Sub-Dimensionen wird die Veränderungsbereitschaft in den identifizierten Reifegradmodellen nicht direkt thematisiert. Veränderungen werden allerdings insbesondere durch die Digitalisierung verursacht (Cisik 2019).

Organisation des MR Abschließend beeinflusst die Organisation des MR die Reife des gesamten Controllings (Keimer et al. 2017; Langmann 2019).

\subsection{Reifegrade}

Die Bestimmung der Reifegrade für das zu entwickelnde Modell folgt den Erkenntnissen der Recherche nach vergleichbaren Modellen und theoretischen Konstrukten rund um Management Reporting und Digitalisierung. Daraus resultieren vier unterschiedliche Reifegrade von „Keine Digitalisierung“ bis „Vollständige Digitalisierung“.

Keine Digitalisierung Auf der niedrigsten Stufe ist eine Situation beschrieben, in der prinzipiell keine der genannten Digitalisierungstechnologien umgesetzt ist. Dadurch können selbst Unternehmen ohne jegliche Adaption dieser Technologien einem konkreten Reifegrad zugeordnet werden, was insbesondere bei der Betrachtung von KMU besonders angebracht erscheint. In der Praxis lässt sich der Reifegrad z. B. daran erkennen, dass Datenbereitstellung ein aufwändiger Prozess ist, bei dem mit möglichst wenigen Ressourcen und durch manuelle Bearbeitung nur grundlegende Daten verfügbar gemacht werden. Es müssen immer wieder Zusammenhänge und Differenzen erklärt werden, da keine Konsistenz vorliegt. Wesentliche KPIs stützen sich auf einfache Berechnungen, häufig zählt statt kontrollierten Daten das „Bauchgefühl“.

Fragmentierte Digitalisierung Es besteht eine Situation, in der erste Ansätze einer Digitalisierung entlang des kompletten Prozesses oder nur in einigen Prozessschritten gegangen wurden. Es werden analytische Erkenntnisse erwartet und nicht nur grundlegende Informationen. Es bestehen Ansätze zu einer effizienten Speicherung und Verarbeitung dieser Daten. Datenqualität und Konsistenz sind verbessert und Prozessschritte wurden bereits automatisiert. Tabellenkalkulationsprogramme sind immer noch ein zentraler Bestandteil des Reportings, aber sie werden teilweise aus Sicht der Performance und der Funktionen ans Limit getrieben.

Ausgeprägte Digitalisierung Bei einer konsequenten Umsetzung von Digitalisierung in MR-Prozessen ist das unternehmerische und organisatorische Umfeld förderlich für Digitalisierungsanstrengungen und Veränderungen. Es werden interne und externe Daten mit einer eigens eingerichteten Architektur verwaltet und abgerufen, z. B. mit einem umfangreichen Data Warehouse. Cloud-Lösungen werden bei Bedarf genutzt, um Lastspitzen abzufangen und moderne Entwicklungen wie Self- 
Service-Reporting und Predictive Analytics zu unterstützen. Das Controlling unterstützt handlungsleitend und strategisch. Der Prozess ist weitgehend automatisiert und die Berichte mit Hilfe technischer Verfahren auch inhaltlich validiert.

Vollständige Digitalisierung Es dominiert ein Umfeld, das die Digitalisierung nicht nur fördert, sondern auch fordert. Dem MR kommt eine zentrale Bedeutung der Unternehmenssteuerung zu. Die Verwendung unterschiedlicher, integrierter Datenquellen ist Standard. Business-Analytics-Verfahren liefern prädiktive und präskriptive Inhalte. KI wird eingesetzt, um neue Erkenntnisse zu erlangen und als interaktiver Sparringspartner den Echtzeit-Zugriff auf Daten zu unterstützen. Das Management Reporting gilt als innovativ, wegweisend und wertschöpfend.

\subsection{Management-Reporting-Prozess-Maturity-Model (MRPMM)}

Kombiniert mit den vier Dimensionen und deren Sub-Dimensionen aus dem vorherigen Abschnitt ergibt sich das inhaltliche ausgearbeitete Reifegradmodell der Digitalisierung der Management-Reporting-Prozesse. Abb. 3 zeigt dieses ManagementReporting-Prozess-Maturity-Model (MRPMM). Abb. 4 zeigt im Anschluss exemplarisch eine Zuordnung von Ausprägungen der Sub-Dimensionen zu Reifegraden am Beispiel der Dimension Datenbereitstellung. Die Darstellung dieser sowie die entsprechenden Darstellungen der weiteren Dimensionen können Onlinematerial 2 entnommen werden.

\section{Digitalisierungsgrad der Management Reporting-Prozesse im Management-Reporting-Prozess-Maturity-Model (MRPMM)}

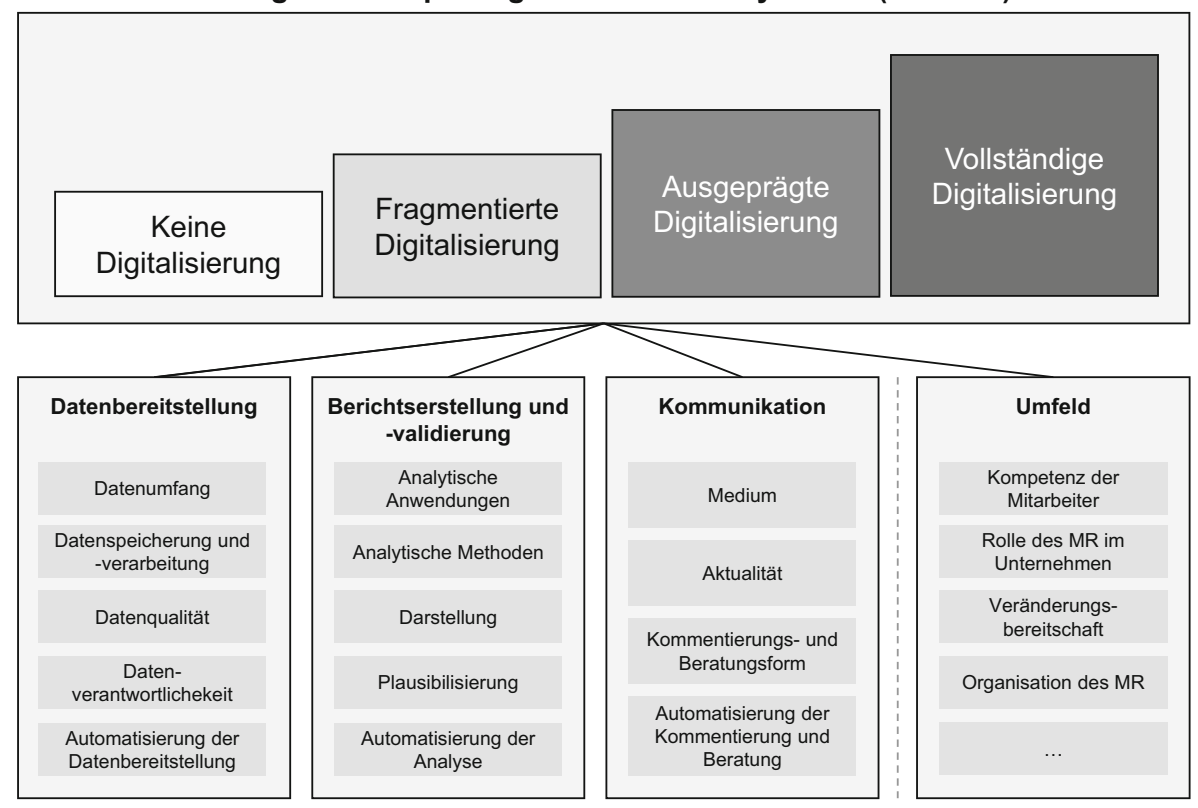

Abb. 3 Das Management-Reporting-Prozess-Maturity-Model (MRPMM) 


\section{Datenbereitstellung}

\begin{tabular}{|c|c|c|c|c|}
\hline Datenumfang & $\begin{array}{l}\text { Wenige und nur interne } \\
\text { sowie strukturierte } \\
\text { Daten werden } \\
\text { verwendet }\end{array}$ & $\begin{array}{l}\text { Volle Nutzung und } \\
\text { Integration aller } \\
\text { strukturierten, internen } \\
\text { Daten }\end{array}$ & $\begin{array}{l}\text { Interne, unstrukturierte } \\
\text { Daten werden } \\
\text { zusätzlich verwendet, } \\
\text { zusätzlich vereinzelte } \\
\text { Nutzung von externen } \\
\text { Daten }\end{array}$ & $\begin{array}{l}\text { Große Mengen von } \\
\text { externen und internen } \\
\text { Daten sowohl in } \\
\text { strukturierter als in } \\
\text { unstrukturierter Form } \\
\text { werden verwendet }\end{array}$ \\
\hline $\begin{array}{l}\text { Daten- } \\
\text { speicherung } \\
\text { und } \\
\text {-verarbeitung }\end{array}$ & $\begin{array}{l}\text { Kein DWH, nur } \\
\text { provisorische oder } \\
\text { keine Datenbanken / } \\
\text { komplizierter und } \\
\text { langsamer Datenzugriff }\end{array}$ & $\begin{array}{l}\text { DWH-Lösung verfügbar } \\
\text { aber nicht } \\
\text { unternehmensweit } \\
\text { umgesetzt }\end{array}$ & $\begin{array}{l}\text { Zentrales DWH in } \\
\text { Kombination mit } \\
\text { Lösungen zu } \\
\text { Speicherung und } \\
\text { Verarbeitung von } \\
\text { unstrukturierten Daten / } \\
\text { Einsatz von Cloud- } \\
\text { Lösungen }\end{array}$ & $\begin{array}{l}\text { Zentrale und hoch } \\
\text { performante Lösungen } \\
\text { zur Speicherung und } \\
\text { Verarbeitung von } \\
\text { sämtlichen Formen von } \\
\text { Daten / Einsatz von } \\
\text { umfassenden Cloud- } \\
\text { Lösungen }\end{array}$ \\
\hline \multirow{2}{*}{ Datenqualität } & $\begin{array}{l}\text { Daten sind } \\
\text { unternehmensweit nicht } \\
\text { konsistent, keine } \\
\text { zentralen Stammdaten }\end{array}$ & $\begin{array}{l}\text { Daten sind größtenteils } \\
\text { konsistent, Stammdaten } \\
\text { werden nur teilweise } \\
\text { zentral gepflegt }\end{array}$ & $\begin{array}{l}\text { Unternehmensweite } \\
\text { Datenkonsistenz und } \\
\text { zentrale } \\
\text { Stammdatenpflege }\end{array}$ & $\begin{array}{l}\text { Unternehmensweite } \\
\text { Datenkonsistenz und } \\
\text { zentrale } \\
\text { Stammdatenpflege }\end{array}$ \\
\hline & $\begin{array}{l}\text { Viele manuelle } \\
\text { Aufbereitungsschritte } \\
\text { sind sind notwendig, } \\
\text { schlechte Datenqualität }\end{array}$ & $\begin{array}{l}\text { Einige } \\
\text { Aufbereitungsschritte } \\
\text { notwendig, nur wenige } \\
\text { Qualitätsprobleme }\end{array}$ & $\begin{array}{l}\text { Kaum zusätzliche } \\
\text { Aufbereitungsschritte } \\
\text { sind notwendig }\end{array}$ & $\begin{array}{l}\text { Keine zusätzliche } \\
\text { Aufbereitung / hohe } \\
\text { Datenqualität / Nutzung } \\
\text { von KI zur Kontrolle }\end{array}$ \\
\hline $\begin{array}{l}\text { Daten- } \\
\text { verantwortlich- } \\
\text { keit }\end{array}$ & $\begin{array}{l}\text { Kein designierter } \\
\text { Verantwortlicher für } \\
\text { Themen rund um das } \\
\text { Datenmanagement }\end{array}$ & $\begin{array}{l}\text { Vereinzelte Themen im } \\
\text { Bereich } \\
\text { Datenmanagement } \\
\text { werden adressiert }\end{array}$ & $\begin{array}{l}\text { Dedizierte } \\
\text { Ansprechpartner zum } \\
\text { Datenmanagement }\end{array}$ & $\begin{array}{l}\text { Umfassende Data } \\
\text { Governance }\end{array}$ \\
\hline \multirow[t]{2}{*}{$\begin{array}{l}\text { Automati- } \\
\text { sierung der } \\
\text { Daten- } \\
\text { bereitstellung }\end{array}$} & $\begin{array}{l}\text { Notwendige } \\
\text { Prozessschritte werden } \\
\text { manuell ausgeführt }\end{array}$ & $\begin{array}{l}\text { Notwendige } \\
\text { Prozessschritte sind } \\
\text { teilweise automatisiert }\end{array}$ & $\begin{array}{l}\text { Notwendige } \\
\text { Prozessschritte sind } \\
\text { größtenteils } \\
\text { automatisiert }\end{array}$ & $\begin{array}{l}\text { Notwendige } \\
\text { Prozessschritte sind } \\
\text { voll automatisiert }\end{array}$ \\
\hline & $\begin{array}{c}\text { Keine } \\
\text { Digitalisierung }\end{array}$ & $\begin{array}{l}\text { Fragmentierte } \\
\text { Digitalisierung }\end{array}$ & $\begin{array}{l}\text { Ausgeprägte } \\
\text { Digitalisierung }\end{array}$ & $\begin{array}{l}\text { Vollständige } \\
\text { Digitalisierung }\end{array}$ \\
\hline
\end{tabular}

Abb. 4 Zuordnung von Ausprägungen der Sub-Dimensionen zu Reifegraden am Beispiel der Dimension Datenbereitstellung

\section{Operationalisierung und Ergebnisinterpretation des Reifegradmodells}

Unter Operationalisierung wird der Prozess verstanden, theoretische Konstrukte, die nicht direkt messbar oder beobachtbar sind, messbar zu machen. Um das zu erreichen, werden Indikatoren bestimmt, über die die erwähnten theoretischen Konstrukte abgebildet werden können (Schmelter 2009).

Dazu wurden alle Sub-Dimensionen betrachtet und geeignete Fragen zur Operationalisierung abgeleitet. Um die Validität zu erhöhen, wurden, wenn möglich und sinnvoll, mehrere Fragen erarbeitet. Bei einem Großteil der Sub-Dimensionen ist allerdings keine Operationalisierung im eigentlichen Sinne notwendig, da direkt nach der relevanten Information gefragt werden kann, ohne Indikatoren verwenden zu müssen. Häufig kann dieser Prozess nach Bedarf vertieft werden. So erfordert beispielsweise die Sub-Dimension Datenumfang keinen kreativen Indikator, es kann gezielt nach relevanten Datenmengen gefragt werden. Entsprechendes gilt für die Kompetenz der Mitarbeiter. Um den letztgenannten Punkt zu vertiefen und herauszufinden, wie die Zukunftsvision zur Kompetenz der Mitarbeiter aussieht, könnte jedoch zusätzlich nach dem Fokus im Recruiting gefragt werden. Beispielhafte 


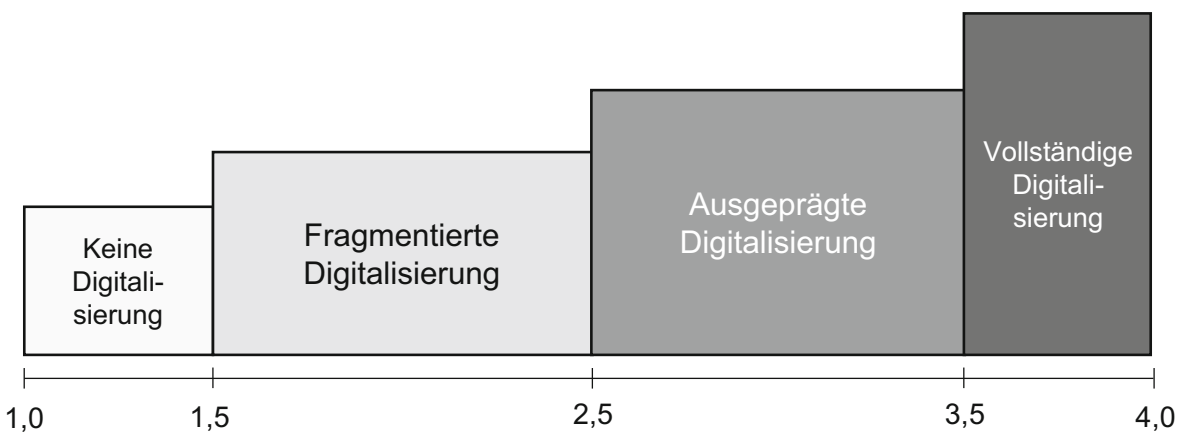

Abb. 5 Bewertungsspanne der Reifegrade im MRPMM

Fragen sind: Welche Kenntnisse besitzen die Verantwortlichen des MR-Prozesses? Welche Kenntnisse stehen beim Recruiting im Mittelpunkt? Weitere beispielhafte Fragen zur Operationalisierung können Onlinematerial 3 entnommen werden.

Durch eine Bewertung der Antworten mit Punkten zwischen 1,0 und 4,0 ergeben sich zunächst Punktesummen je Sub-Dimension. Diese können wiederum individuell gewichtet werden. Es ergibt sich durch gewichtete Summierung ein Reifegrad je Dimension. Auch für die Dimensionen können individuelle Gewichte vergeben werden. So kann beispielsweise durch ein niedriges Gewicht der Dimension „Umfeld“ berücksichtigt werden, dass diese nur indirekt auf den Digitalisierungsgrad des MR wirkt (vgl. Abschn. 4.3.4). Unternehmen können durch die Einzeldarstellung vor der gewichteten Zusammenführung auch gezielt Schwachstellen oder bereits hoch digitalisierte Bereiche identifizieren und konkrete Handlungen ableiten, anstatt nur einen Durchschnittswert zu betrachten. Um eine Vergleichbarkeit unterschiedlicher Unternehmen herzustellen, müssen die Fragen und Gewichte identisch sein. Um aber z. B. unternehmensintern den Fortschritt über die Zeit oder verschiedene Abteilungen betrachten zu können, besteht die Möglichkeit, dass Unternehmen individuelle Gewichtungsschlüssel der (Sub-)Dimensionen festlegen. Der berechnete gewichtete Durchschnittswert aller Antworten ergibt dann - so wie die Einzelergebnisse je Dimension - eine Zuordnung auf die Reifegrade gemäß der Darstellung in Abb. 5.

Ein Ergebnis unter oder gleich 1,5 signalisiert, dass der Digitalisierungsstand der MR-Prozesse insgesamt dem Reifegrad „Keine Digitalisierung“ zugeordnet werden kann. Für dieses Ergebnis ist zu erwarten, dass ein Großteil der Antworten bei den Werten 1,0 oder 1,5 liegt, welchen die Antwort zugeordnet ist, die mit der niedrigsten Ausprägung der Sub-Dimensionen korrespondiert. Falls es einzelne Ausreißer gab, so wird dies durch eine Betrachtung der Ergebnisse der einzelnen Sub-Dimensionen deutlich.

Die beiden „Rand-Reifegrade“ umfassen eine geringere Punktespanne, um deutlich zu machen, dass sowohl ,keine“ als auch eine ,vollständige“ Digitalisierung in der Praxis eher die Ausnahme sind (vgl. zur Digitalisierung Büchel et al. (2021), Deutsche Telekom (2020) oder Grzymek und Wintermann (2020)). Insbesondere die vollständige Digitalisierung kann auch nur unter der Maßgabe einer Einschätzung aktueller technologischer Entwicklungen betrachtet werden, da die hier vorgestellten Digitalisierungstechnologien zum Zeitpunkt der Modellerstellung angemessen 
scheinen, im Verlaufe der Zeit der maximal erreichbare Stand jedoch durch neue technologische Entwicklungen weiter verschoben werden kann.

\section{Zusammenfassung}

Die große Bandbreite des Themenkomplexes „Digitalisierung“ in Kombination mit kontinuierlicher Weiterentwicklung und neuen Möglichkeiten macht es besonders notwendig, die eigene Position sowie mögliche Verbesserungspotentiale zu kennen. Dies ist grundlegend für eine erfolgreiche (Prozess-)Weiterentwicklung. Der Bereich Management Reporting im Controlling ist hiervon im Besonderen betroffen.

Das vorliegende Reifegradmodell zeigt dabei auf, welche Dimensionen und Reifegrade für eine Betrachtung des Status quo verwendet werden. Eine mögliche Einordnung des Ergebnisses kann mit Hilfe von Benchmarks erfolgen (de Bruin et al. 2005). Studien zur Unternehmensgröße (BITKOM 2018) oder Branche (Becker et al. 2013) und deren Standpunkt bei dem Thema Digitalisierung deuten an, dass eine Korrelation zwischen Unternehmensgröße, Branche und vergleichbaren Faktoren und dem Digitalisierungsgrad besteht. Je kleiner das Unternehmen, je weniger digitalisiert sind in der Regel die Prozesse.

Ein Blick auf die identifizierten, wesentlichen Technologien deutet darauf hin, dass insbesondere Cloud-Computing-Ansätze (z. B. Software-as-a-Service) und RPA-Optionen für den Mittelstand einen Einstieg in die Optimierung der MRProzesse sein können. So könnten Fortschritte bei der Automatisierung gemacht werden und die Nutzung von großen Datenmengen oder fortschrittlicher AnalyseSoftware wird ggf. ohne große Investitionen möglich.

Eine iterative Weiterentwicklung des MRPMM kann anhand einer ausgedehnten Evaluation und einer empirischen Validierung des Modells erfolgen. Wie beschrieben ist zudem eine regelmäßige Pflege des Modells im Hinblick auf neue technologische Möglichkeiten notwendig, um eine fortwährende Relevanz zu gewährleisten.

Zusatzmaterial online Zusätzliche Informationen sind in der Online-Version dieses Artikels (https://doi. org/10.1365/s40702-021-00787-z) enthalten.

Funding Open Access funding enabled and organized by Projekt DEAL.

Open Access Dieser Artikel wird unter der Creative Commons Namensnennung 4.0 International Lizenz veröffentlicht, welche die Nutzung, Vervielfältigung, Bearbeitung, Verbreitung und Wiedergabe in jeglichem Medium und Format erlaubt, sofern Sie den/die ursprünglichen Autor(en) und die Quelle ordnungsgemäß nennen, einen Link zur Creative Commons Lizenz beifügen und angeben, ob Änderungen vorgenommen wurden.

Die in diesem Artikel enthaltenen Bilder und sonstiges Drittmaterial unterliegen ebenfalls der genannten Creative Commons Lizenz, sofern sich aus der Abbildungslegende nichts anderes ergibt. Sofern das betreffende Material nicht unter der genannten Creative Commons Lizenz steht und die betreffende Handlung nicht nach gesetzlichen Vorschriften erlaubt ist, ist für die oben aufgeführten Weiterverwendungen des Materials die Einwilligung des jeweiligen Rechteinhabers einzuholen.

Weitere Details zur Lizenz entnehmen Sie bitte der Lizenzinformation auf http://creativecommons.org/ licenses/by/4.0/deed.de. 


\section{Literatur}

Aho M (2009) A capability maturity model for corporate performance management. An empirical study in large Finnish manufacturing companies. In: Proceedings from the eBRF - A Research Forum to Understand Business in Knowledge Society Jyväskylä, Finland

Becker J, Knackstedt R, Pöppelbuß J (2009) Entwicklung von Reifegradmodellen für das IT-Management. Wirtschaftsinformatik 51:249-260. https://doi.org/10.1007/s11576-009-0167-9

Becker W, Ulrich P, Vogt M (2013) Digitalisierung im Mittelstand - Ergebnisbericht einer Online-Umfrage. Bamberger Betriebswirtschaftliche Beiträge, Bd. 192. Otto-Friedrich-Universität Bamberg, Bamberg

BITKOM (2018) Digitalisierung der Wirtschaft. https:/www.bitkom.org/sites/default/files/file/import/ Bitkom-Charts-Digitalisierung-der-Wirtschaft-06-06-2018-final.pdf. Zugegriffen: 16. Jan. 2021

vom Brocke J, Simons A, Niehaves B, Riemer K, Plattfaut R, Cleven A (2009) Reconstructing the giant: on the importance of rigour in documenting the literature search process. In: Proceedings of the 17 th European Conference on Information Systems (ECIS) Verona, Italy

de Bruin T, Rosemann M, Freeze R, Kulkarni U (2005) Understanding the main phases of developing a maturity assessment model. In: ACIS 2005 Proceedings Sydney, Australia

Büchel J, Demary V, Goecke H, Rusche C (2021) Digitalisierung der Wirtschaft in Deutschland Digitalisierungsindex 2020. https:/www.de.digital/DIGITAL/Redaktion/DE/Digitalisierungsindex/ Publikationen/publikation-download-Langfassung-digitalisierungsindex-2020.pdf. Zugegriffen: 16. Juli 2021

Burrus D, Hood T (2020) Big data, artificial intelligence, RPA and cloud top the list of profession's technology hard trends for 2020. https://blionline.org/big-data-artificial-intelligence-rpa-and-cloud-topthe-list-of-professions-technology-hard-trends-for-2020/. Zugegriffen: 16. Jan. 2021

Cates JE, Gill SS, Zeituny N (2005) The ladder of business intelligence (LOBI): a framework for enterprise IT planning and architecture. Int J Bus Inf Syst 1:220-238. https://doi.org/10.1504/ijbis.2005.007408

Chamoni P, Gluchowski P (2004) Integrationstrends bei Business-Intelligence-Systemen. Wirtschaftsinformatik 46:119-128. https://doi.org/10.1007/bf03250931

Chamoni P, Gluchowski P (2017) Business analytics-state of the Art. Control Manag Rev 61:8-17. https://doi.org/10.1007/s12176-017-0030-6

Cisik A (2019) Veränderungen in Unternehmen. Ergebnisse einer empirischen Studie. http://www. cisikconsulting.de/wp-content/uploads/2019/09/Gestaltung-und-Erleben-von-Veränderungen_ Management-Summary_September-2019.pdf. Zugegriffen: 16. Jan. 2021

De Massis A, Audretsch D, Uhlaner L, Kammerlande N (2018) Innovation with limited resources: management lessons from the German mittelstand. J Prod Innov Manag 35:125-146. https://doi.org/10. 1111/jpim. 12373

Deutsche Telekom (2020) Digitalisierungsindex Mittelstand 2020/2021 - Der digitale Status quo des deutschen Mittelstands. https://www.digitalisierungsindex.de/wp-content/uploads/2020/12/Telekom_ Digitalisierungsindex_2020_GESAMTBERICHT.pdf. Zugegriffen: 16. Juli 2021

Dursun D (2019) Big Data für das Management. Control Manag Rev 63:46-52. https://doi.org/10.1007/ s12176-018-0104-0

Fasel D, Meier A (2016) Was versteht man unter Big Data und NoSQL? In: Fasel D, Meier A (Hrsg) Big Data - Grundlagen, Systeme und Nutzungspotenziale. Springer Vieweg, Wiesbaden, S 3-16 https:// doi.org/10.1007/978-3-658-11589-0_1

Felden C (2019) Business analytics. https://www.enzyklopaedie-der-wirtschaftsinformatik.de/wienzyklopaedie/lexikon/daten-wissen/Business-Intelligence/Analytische-Informationssysteme-Methoden-der-/Business-Analytics/index.html. Zugegriffen: 16. Jan. 2021

FINANCE (2019) CFO Panel: Umfrage Herbst 2019. https://www.finance-magazin.de/fileadmin/PDF/ Panels/FINANCE-CFO-Panel-02-2019.pdf. Zugegriffen: 16. Jan. 2021

Forrester (2018) Harness RPA to optimize customer outcomes. https://dfe.org.pl/wp-content/uploads/2018/ 12/Forrester_Harness-RPA.pdf. Zugegriffen: 5. Juli 2021

Friedl G (2019) Künstliche Intelligenz im Controlling. Controlling 31:35-38. https://doi.org/10.15358/ 0935-0381-2019-5-35

Fürber C (2016) Data quality management with semantic technologies. Springer, Wiesbaden https://doi. org/10.1007/978-3-658-12225-6

Gräf J, Isensee J, Mehanna W (2014) Management Reporting: Aufgaben, Konzeption und Umsetzung. In: Klein A, Gräf J (Hrsg) Reporting und Business Intelligence. Berichte klar gestalten, effizient erstellen, prägnant kommentieren, 2. Aufl. Haufe, München, S 25-42 
Grzymek V, Wintermann O (2020) Wie digital sind die Unternehmen in Deutschland? Ergebnisse einer repräsentativen Befragung unter Erwerbstätigen. Bertelsmann Stiftung, Gütersloh https://doi.org/10. $11586 / 2020001$

Halper F, Krishnan K (2013) TDWI big data maturity model guide. https://tdwi.org/whitepapers/2013/10/ tdwi-big-data-maturity-model-guide.aspx?tc=page0. Zugegriffen: 16. Jan. 2021

Hevner AR, March ST, Park J, Ram S (2004) Design science in information systems research. MISQ 28:75-105. https://doi.org/10.2307/25148625

Holinski B (2014) Berichte gekonnt kommentieren: Was Controller von Twitter lernen können. In: Klein A, Gräf J (Hrsg) Reporting und Business Intelligence. Berichte klar gestalten, effizient erstellen, prägnant kommentieren, 2. Aufl. Haufe, München, S 59-66

Hölzle K, Petzolt S, Kullik O, Gerhardt F (2019) Reifegradmessung zur digitalen Transformation von KMU. Universität Potsdam, Potsdam https://doi.org/10.13140/RG.2.2.28402.66242

Isensee J, Emler M, Schönherr M, Goede L (2019) Digitale Transformation im Reporting. Durch Digitalisierung Nutzen und Effizienz des Reportings steigern. https://www.horvath-partners.com/ de/media-center/studien/digitale-transformation-im-reporting-durch-digitalisierung-nutzen-undeffizienz-des-reportings-steigern/. Zugegriffen: 16. Jan. 2021

Kamprath N (2011) Einsatz von Reifegradmodellen im Prozessmanagement. HMD 48:93-102. https://doi. org/10.1007/bf03340648

Keimer I, Zorn M, Gisler MG, Fallegger M (2017) Dimensionen der Digitalisierung im Controlling. Grundlagen und Denkanstösse zur Selbstanalyse und Weiterentwicklung. Expert Focus 11:827-831

Kirste M, Schürholz M (2019) Entwicklungswege zur KI. In: Wittpahl V (Hrsg) Künstliche Intelligenz - Technologie, Anwendung, Gesellschaft. Springer Vieweg, Berlin, S 21-35 https://doi.org/10.1007/ 978-3-662-58042-4

Koß R (2016) Ein Reifegradmodell für das digitale Controlling. Control Manag Rev 60:32-39. https://doi. org/10.1007/s 12176-016-0092-X

KPMG (2018) Digitalisierung im Rechnungswesen 2018. https://hub.kpmg.de/digitalisierung-imrechnungswesen-2018. Zugegriffen: 16. Jan. 2021

Langmann C (2019) Digitalisierung im Controlling. Springer Gabler, Wiesbaden https://doi.org/10.1007/ 978-3-658-25017-1

Langmann C, Turi D (2020) Robotic Process Automation (RPA) - Digitalisierung und Automatisierung von Prozessen: Voraussetzungen, Funktionsweise und Implementierung am Beispiel des Controllings und Rechnungswesens. Springer Gabler, Wiesbaden https://doi.org/10.1007/978-3-658-28299-8

Losbichler H, Eisl C, Plank T (2016) Neue Visualisierungsformen auf dem Prüfstand. Control Manag Rev 60:46-53. https://doi.org/10.1007/s12176-016-0010-2

Marx F, Wortmann F, Mayer JH (2012) Ein Reifegradmodell für Unternehmenssteuerungssysteme. Wirtschaftsinformatik 54:189-204. https://doi.org/10.1007/s11576-012-0325-3

Peffers K, Tuunanen T, Rothenberger MA, Chatterjee S (2007) A design science research methodology for information systems research. J Manag Inf Syst 24:45-77. https://doi.org/10.2753/mis07421222240302

Pöppelbuß J, Röglinger M (2011) What makes a useful maturity model? A framework of general design principles for maturity models and its demonstration in business process management. In: Proceedings of the 19th European Conference on Information Systems (ECIS) Helsinki, Finland

Schäffer U, Weber J (2016) Die Digitalisierung wird das Controlling radikal verändern. Control Manag Rev 60:6-17. https://doi.org/10.1007/s12176-016-0093-9

Schmelter R (2009) Der Einfluss von Management auf Corporate Entrepreneurship. Gabler, Wiesbaden https://doi.org/10.1007/978-3-8349-9452-3_5

Schön D (2012) Planung und Reporting im Mittelstand - Grundlagen, Business Intelligence und Mobile Computing. Springer Gabler, Wiesbaden https://doi.org/10.1007/978-3-8349-3604-2_4

Schön D (2018) Planung und Reporting im BI-gestützten Controlling - Grundlagen, Business Intelligence, Mobile BI und Big-Data-Analytics, 3. Aufl. Springer Gabler, Wiesbaden https://doi.org/10.1007/9783-658-19963-0_1

Schulze R, Watling L (2013) Reifegrade von Management-Informationssystemen. Control Mag 3:15-22

Shmueli G, Koppius OR (2011) Predictive analytics in information systems research. MISQ 35:553-572. https://doi.org/10.2307/23042796

Spruit M, Sacu C (2015) DWCMM: the data warehouse capability maturity model. J Univers Comput Sci 21:1508-1534

Statistisches Bundesamt (Destatis) (2020) Unternehmen, Tätige Personen, Umsatz und weitere betriebs- und volkswirtschaftliche Kennzahlen: Deutschland, Jahre, Unternehmensgröße. https://wwwgenesis.destatis.de/genesis/online. Zugegriffen: 16. Jan. 2021 
Tan CS, Sim YW, Yeoh W (2011) A maturity model of enterprise business intelligence. Commun IBIMA. https://doi.org/10.5171/2011.417812

Taschner A (2013) Management Reporting für Praktiker. Springer Gabler, Wiesbaden https://doi.org/10. 1007/978-3-658-01111-6

Taschner A (2019) Management reporting und behavioral accounting. Springer Gabler, Wiesbaden https:// doi.org/10.1007/978-3-658-23492-8

Tavallaei R, Shokohyar S, Moosavi SM, Sarfi Z (2015) Assessing the evaluation models of business intelligence maturity and presenting an optimized model. Int J Manag Account Econ 2:1005-1019

Wagner KP (2012) Selbstbewertung des Reifegrads des IT-Einsatzes in KMU. Wirtschaftsinf Manag 4:34-43

Webster J, Watson RT (2002) Analyzing the past to prepare for the future: writing a literature review. MIS Q 26:xiii-xxiii

Weichel P, Herrmann J (2016) Wie Controller von Big Data profitieren können. Control Manag Rev 60:7-15. https://doi.org/10.1007/s12176-016-0012-0

Willcocks LP, Lacity M, Craig A (2015) The IT function and robotic process automation. The London School of Economics and Political Science, London 